\title{
Molecular network-based analysis of the mechanism of liver injury induced by volatile oils from Artemisiae argyi folium
}

\author{
Hongjie Liu ${ }^{1+}$, Sha Zhan ${ }^{1 \dagger}$, Yan Zhang ${ }^{2}$, Yan Ma², Liang Chen ${ }^{1}$, Lingxiu Chen ${ }^{1}$, Hanqiu Dong ${ }^{1}$, Min Ma ${ }^{1 *}$
} and Zhe Zhang ${ }^{2^{*}}$ (D)

\begin{abstract}
Background: Volatile oils from Artemisiae argyi folium (VOAAF) is reported with hepatotoxicity, but the underlying mechanism is still unclear.

Methods: In the present study this molecular mechanism was explored with the Ingenuity Pathway Analysis (IPA). The chemical components of the VOAAF were searched in the database, and their target proteins were all identified in the PubChem, while drug-induced liver injury (DILI) genes were searched in the PubMed gene databases. The molecular network of protein targets for VOAAF and DILI genes was built with the IPA. The canonical pathways between the 2 networks were compared to decipher the molecular mechanisms of the liver injury induced by VOAAF.

Results: There were 159 target proteins for VOAAF and 338 genes related to DILI identified, which were further analyzed in the IPA. The canonical pathway comparison showed that VOAAF and DILI both worked on aryl hydrocarbon receptor (AHR), lipopolysaccharide (LPS)/interleukin 1 (IL-1) mediated inhibition of retinoid X receptor (RXR) function, pregnane $X$ receptor (PXR)/RXR activation, xenobiotic metabolism, peroxisome proliferator-activated receptor (PPAR), hepatic cholestasis, farnesoid X receptor (FXR)/RXR activation, and glucocorticoid receptor.
\end{abstract}

Conclusion: VOAAF-induced liver injury may be involved in many pathways in which the AHR signaling and LPS/IL1 mediated inhibition of RXR function pathways could be the most vital.

Keywords: Volatile oils from Artemisiae argyi folium, Chinese herbal drugs, Drug-induced liver injury, Bioinformatics

\section{Background}

The concept of bioinformatics was for the first time introduced at the forum of Information Theory in Biology in 1956 in Gatlinburg, Tennessee, USA [1, 2]. Bioinformatics, a discipline, based on the study of the biological data, applies computer science, statistics, mathematics, and engineering to analyze and interpret biological data $[3,4]$. Bioinformatics analysis technology, which can integrate multiple data and help us to better understand biological processes in organic life, has become an essential way for life sciences studies. With

\footnotetext{
* Correspondence: tmamin@jnu.edu.cn; zhe_zhang@hotmail.com

${ }^{\dagger}$ Equal contributors

'Department of Traditional Chinese Medicine, Jinan University, Guangzhou

510632, China

${ }^{2}$ Shandong University of Traditional Chinese Medicine, Jinan 250355, China
}

the progress and development of modern biological technology, bioinformatics has been applied to the research work in TCM combined with other biological disciplines. Chinese herbal drugs have many compositions and various pharmacological effects. In order to understand the underlying mechanism, currently bioinformatics has been applied to study the functions and biological properties of Chinese herbal drugs [5].

For example, anti-tumor mechanism of Artemisia annua was studied by using on bioinformatics, and the results showed that the anti-tumor effect of Artemisia annua may be associated with regulating lipid metabolism of tumor cells, releasing energy, reducing the rate of tumor cell division and accelerating tumor cell apoptosis [6]. Also bioinformatics was applied to study the antiAlzheimer's herbal medicines, and it was found that 
some herbal medicines were promising for treating Alzheimer's disease [7]. Besides, screening using modular analysis bioinformatics techniques found 6 core functional modules for tumor-associated macrophages (TAMs) that contain 46 total genes [8].

Artemisiae argyi folium, the dry leaf of Artemisia Argyi Lévl. et Vant, is one of the commonly-used Chinese herbal drugs. According to theory of traditional Chinese medicine (TCM), Artemisiae argyi folium enters liver, spleen and kidney meridians, with warm property, pungent and bitter flavors and of slightly toxicity [9]. Reportedly, oral administration of 20-30 g of the crude drugs would result in acute liver injury or even death [10]. The chemical components of Artemisiae argyi folium mainly include volatile oils, flavonoid, tannins, triterpenes and polysaccharides. Volatile oils from Artemisiae argyi folium (VOAAF) have strong pharmacological activities and toxicity. The pharmacological activities of VOAAF include anti-inflammatory, antimicrobial, antiasthma, analgesic, antivirus, antioxidant, antitumor and immunomodulatory effects [11-14]. Although the toxicity of plants may also be the presence of heavy metals and secondary metabolites $[15,16]$, the hepatotoxicity of Artemisiae argyi folium is mainly from its volatile oils $[17,18]$, while the underlying mechanism of VOAAF leading to acute liver injury has not been clarified so far.

In the present study, drug-induced liver injury (DILI) and VOAAF were investigated through bioinformatics by searching public databases such as GenBank, PubChem, and China National Knowledge Infrastructure (CNKI).

\section{Methods}

\section{DILI-related gene data}

DILI related genes were searched in NCBI Gene database (http://www.ncbi.nlm.nih.gov/gene), which integrates information from a wide variety of species. A record may include nomenclature, reference sequences, maps, pathways, variations, phenotypes, and links to genome-, phenotype-, and locus- specific resources worldwide. We searched the Gene database (until June 1, 2016) with "drug induced liver injury" or "DILI" as keywords, DILI genes were targeted for Homo sapiens. (Additional file 1: Table S1).

\section{VOAAF-related target proteins}

The target proteins of the VOAAF were searched in PubChem (http://pubchem.ncbi.nlm.nih.gov/) (until June 1,2016 ), which is a public database on biological activities of small molecules and small interfering RNAs (siRNAs), hosted by the National Institutes of Health of American, consisting of three databases: PubChem Compound, PubChem Bioassay, and PubChem Substance. All the targeted proteins related to the active compounds in
Chinese herbal drugs can be obtained from PubChem. After searching in the Grand Dictionary of Chinese Medicine [6], Chinese Materia Medical [7], Pharmacopoeia of the People's Republic of China [9] and CNKI, the active compounds of the VOAAF were determined, and then all the determined compounds were searched in PubChem Compound. Because of the bio-information could be cross-referenced to other NCBI databases [8], the target proteins of active compounds that were also searched in PubChem. The protein category was limited to Homo sapiens (Additional file 1: Table S2).

\section{Networks built and analysis}

Related DILI genes were input into the IPA software online (IPA, http://www.ingenuity.com), to build the molecular gene network (Fig. 1). Similarly, the VOAAF target proteins were input into IPA software online (IPA, http://www.ingenuity.com). The molecules input into IPA software were termed as "focus molecules". IPA generated focus molecules protein-protein interaction (PPI) as a set of networks based on different bio-functions. Molecules were represented as nodes, and the biological relationship between two nodes is represented as an edge (line). All the edges were supported by at least 1 reference from the literature, textbooks, or from canonical information stored in the Ingenuity Knowledge Base. Nodes were displayed using various shapes that represent the gene product. The networks were evaluated by the scores, which were generated through calculation in the IPA software and represented the significance of the molecules in the network. To investigate the mechanism of DILI induced by the VOAAF, a canonical pathway analysis was performed using the IPA compare module. Through Fisher's exact test, the significance of the association between focus molecules and the canonical pathways were determined in the IPA software.

\section{Results \\ DILI-related gene networks and VOAAF-related target protein networks}

In this study, genes related to DILI were determined and the mechanism of the VOAAF-induced liver injury were explored by using a complex bioinformatics system correlated with GenBank database and target proteins. In the GenBank database 338 genes were identified (Additional file 1: Table S1) involved in DILI. By using the Ingenuity Pathway Analysis (IPA) kit (QIAGEN, Shanghai, China), the DILI molecular network was determined (Fig. 2) by importing the associated information for the 338 genes. As shown in Fig. 2, the most related pathways to DILI gene were overlapped. The main functions of the DILI molecular network were cellular function and maintenance, cell death and survival, cell-to-cell signaling and interaction. 


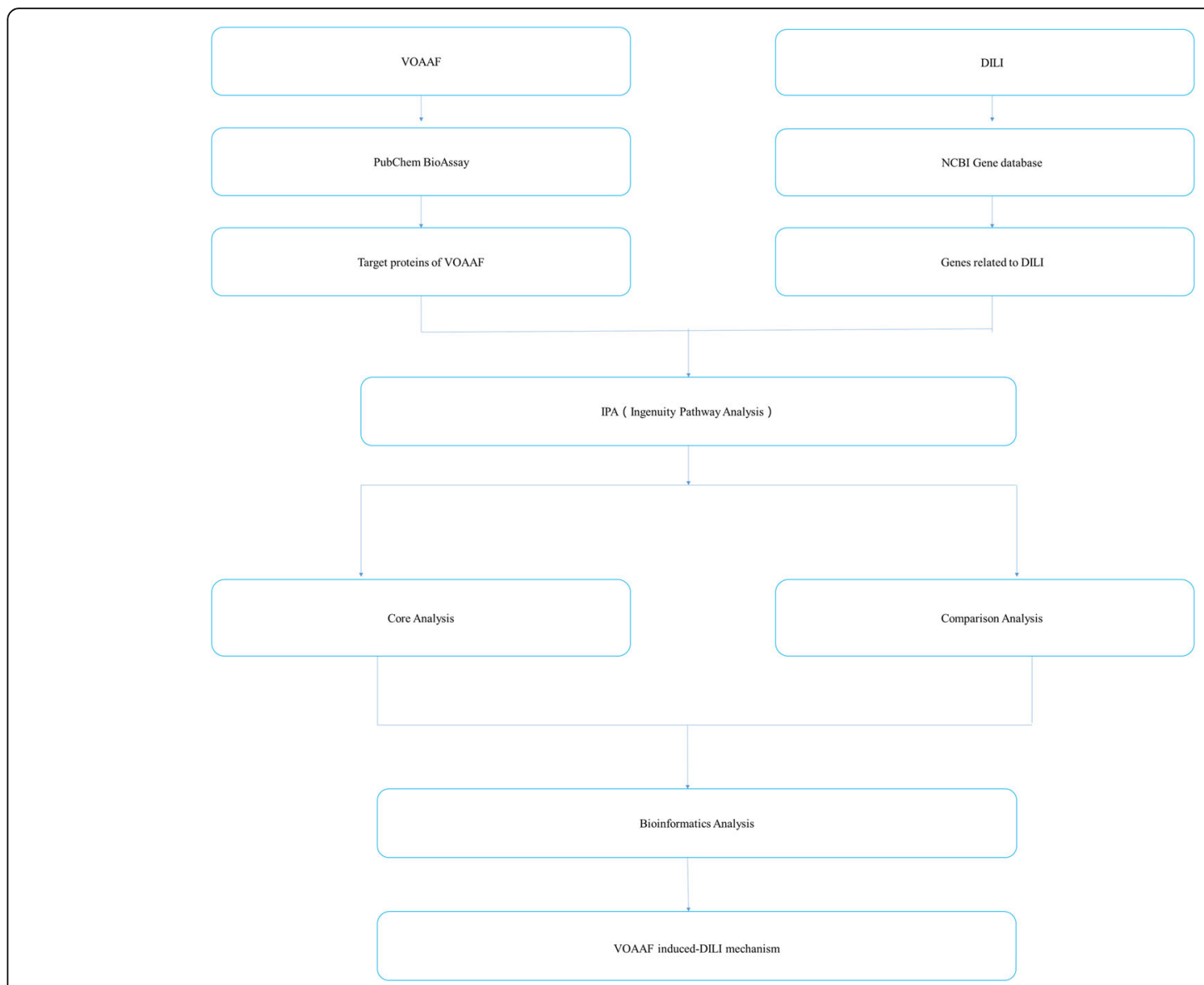

Fig. 1 Frame diagram of the current research

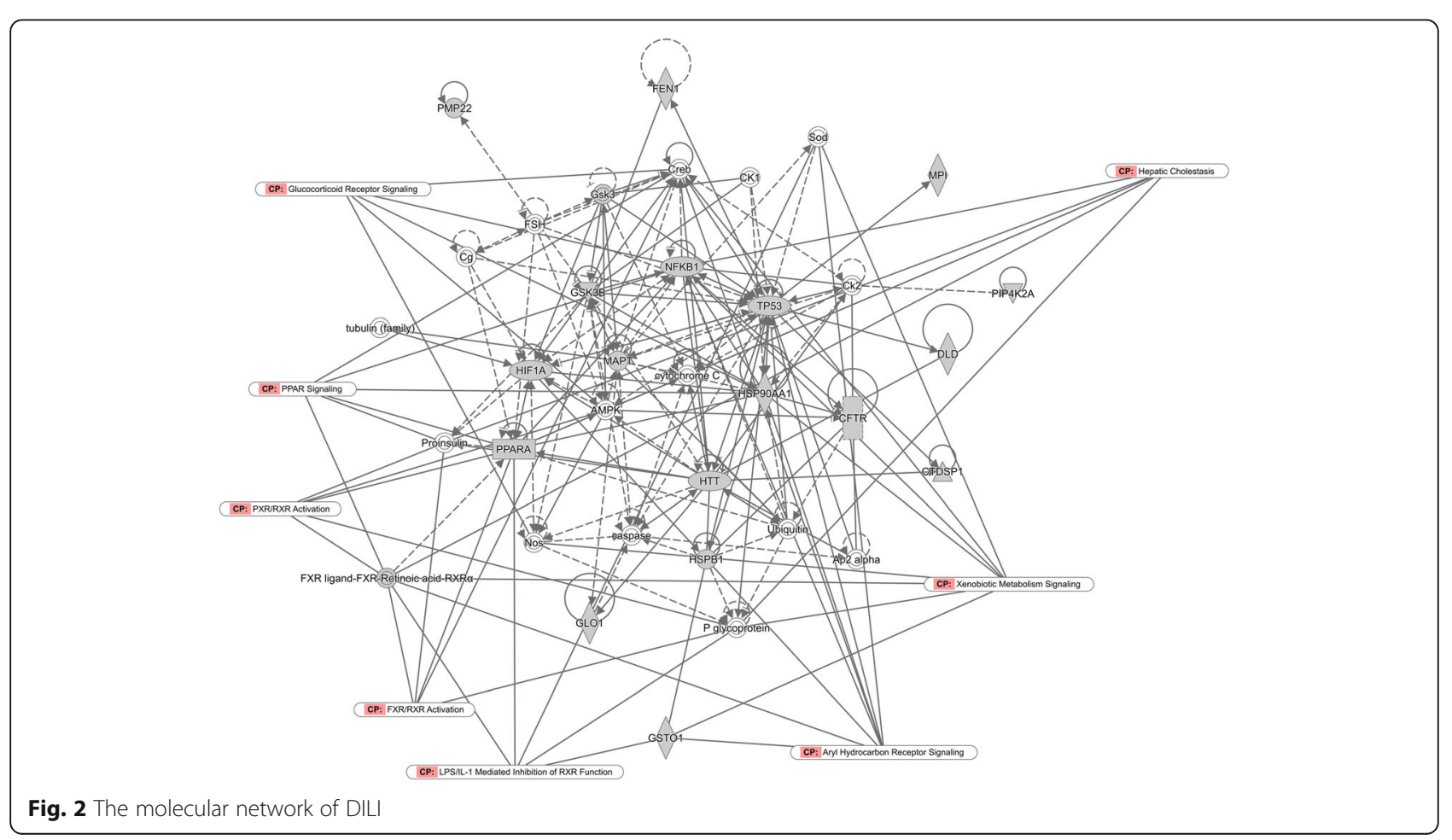


After searched in the PubChem database, information on the drug targets of the VOAAF were listed in Additional file 2: Table S2. The Gen Info Identifier (GI) numbers for each protein were imported into the IPA software, and the PPI networks of VOAAF were constructed (Fig. 3). As shown in Fig. 3, the most related pathways to VOAAF proteins were overlapped. The VOAAF target proteins were involved in metabolic disease, neurological disease, organismal injury and abnormalities.

From the comparison analysis it was found that both the DILI and VOAAF networks, as well as the functions of related target proteins were related on the cell death and survival, cell cycle, lipid metabolism, small molecule biochemistry, vitamin and mineral metabolism.

\section{Diseases and toxic functions}

The functional analysis identified the toxic functions and/or diseases that were most significant to the data set. Proteins from the data set that met the $P$ value threshold of 0.05 (Fisher's exact test) and were associated with toxic functions and/or diseases in the Ingenuity Pathway Analysis Knowledge Database were analyzed. From the IPA analysis (Fig. 4), it was found that the target proteins of VOAAF involved in the diseases and toxic functions are as follows: failure of heart, chronic heart failure, cell death of kidney cell lines, damage of liver, apoptosis of kidney cell lines, injury of liver, hepatocellular carcinoma, necrosis of liver, inflammation of liver, viral hepatitis, chronic viral hepatitis and proliferation of liver cells.

\section{The canonical pathway of DILI and VOAAF networks analyzed by IPA}

Canonical pathway analysis identified the pathways most significant to the data set, based on two parameters: (1) a ratio of the number of proteins from the data set that map to the pathway divided by the total number of proteins that map to the canonical pathway and (2) a $P$ value calculated with Fisher's exact test determining the probability that the association between the proteins in the dataset and the canonical pathway is explained by chance alone. To elucidate the mechanism, we chose the pathways with molecules that were both with large sum in target proteins of VOAAF and DILI genes (Fig. 5). The core analysis of the canonical pathway indicated that target proteins of VOAAF and DILI genes both involved in the pathways of aryl hydrocarbon receptor (AHR) signaling, lipopolysaccharide (LPS)/interleukin 1 (IL-1) mediated inhibition of retinoid X receptor (RXR) function, pregnane $\mathrm{X}$ receptor (PXR)/RXR activation, xenobiotic metabolism signaling, peroxisome proliferator-activated receptor (PPAR) signaling, hepatic cholestasis, farnesoid $\mathrm{X}$ receptor (FXR)/RXR activation, and glucocorticoid receptor signaling. Those pathways are all with high ratio and $P$ value according to the test.

With the comparison analysis (Fig. 6), it was found that the DILI and VOAAF networks had common

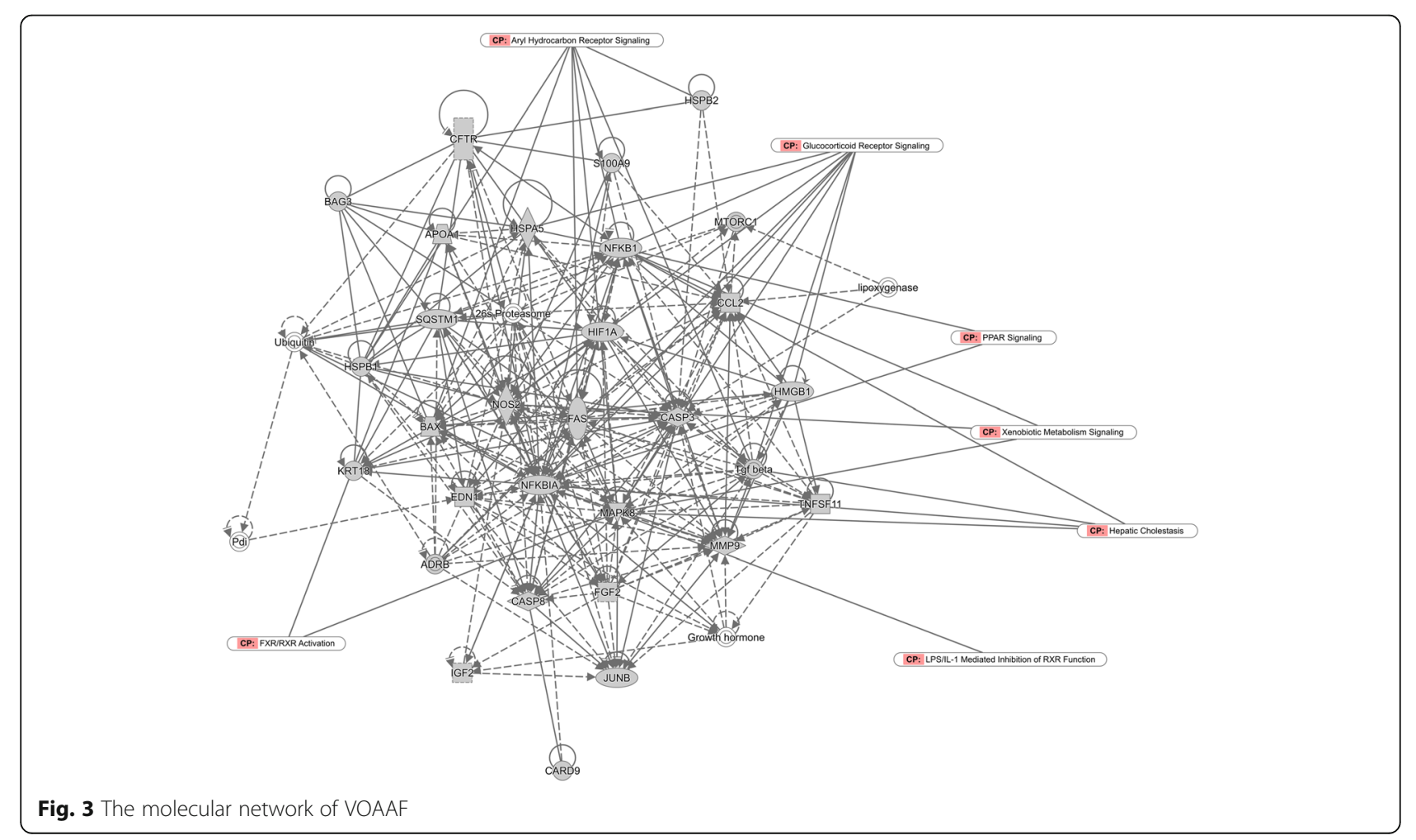




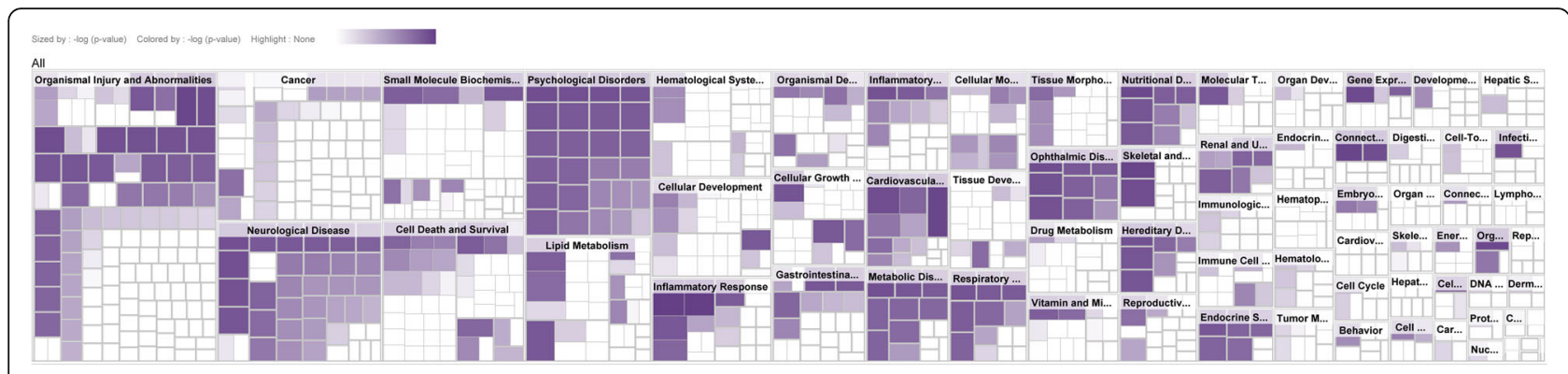

Fig. 4 The heat map of diseases and toxic functions related to target proteins of VOAAF

pathways in gene expression, lipid metabolism, molecular transport, small molecule biochemistry. The aryl hydrocarbon receptor signaling, LPS/IL-1 mediated inhibition of RXR function were also involved.

\section{Discussion}

According to network biology, life is a complex network which is interacted with various molecules. The occurrence and development of disease relates to a series of interacting genes or proteins, and the disease phenotype reflects the different pathological biological processes in a complex network of interactions [19]. Chinese herbal drugs have many compositions and various pharmacological effects. Bioinformatics, based on the analysis of molecular network, could reveal the biological significance of the data contained through acquiring, processing, storage, retrieval and analysis the biological experimental data $[20,21]$. Based on the systems biology data, the advanced bioinformatics analysis technology has become an important means to explore mechanism and pharmacological effect of Chinese herbal drugs [22]. We hope that through analysis of molecular network this research can provide a basis for a better understanding of the molecular mechanisms of the liver injury induced by VOAAF.

The Liver Disease Group of the Chinese Medical Association defined the liver injury caused by drug hepatotoxicity as DILI [23], which includes the liver injury caused by the drug itself and/or its metabolites in the process of drug use or liver injury caused by drug allergy reaction [24], collectively called drug-induced hepatitis [25]. The onset of DILI is a complex process, and its pathogenesis has become a research hotspot in recent years. The pathogenesis of DILI in recent years is mainly considered as follows: the intrinsic hepatotoxicity mechanism, the immune idiosyncratic hepatotoxicity

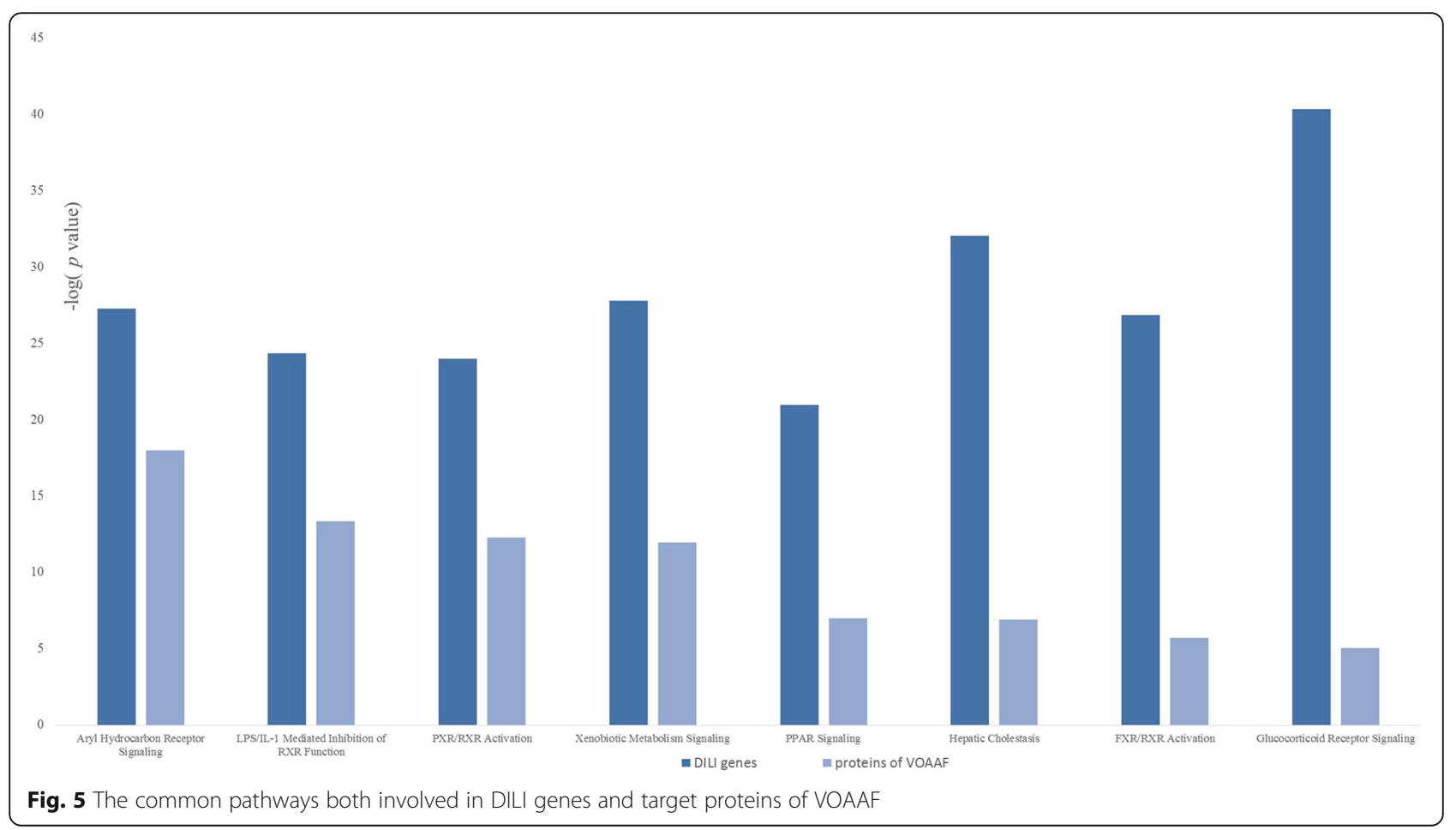




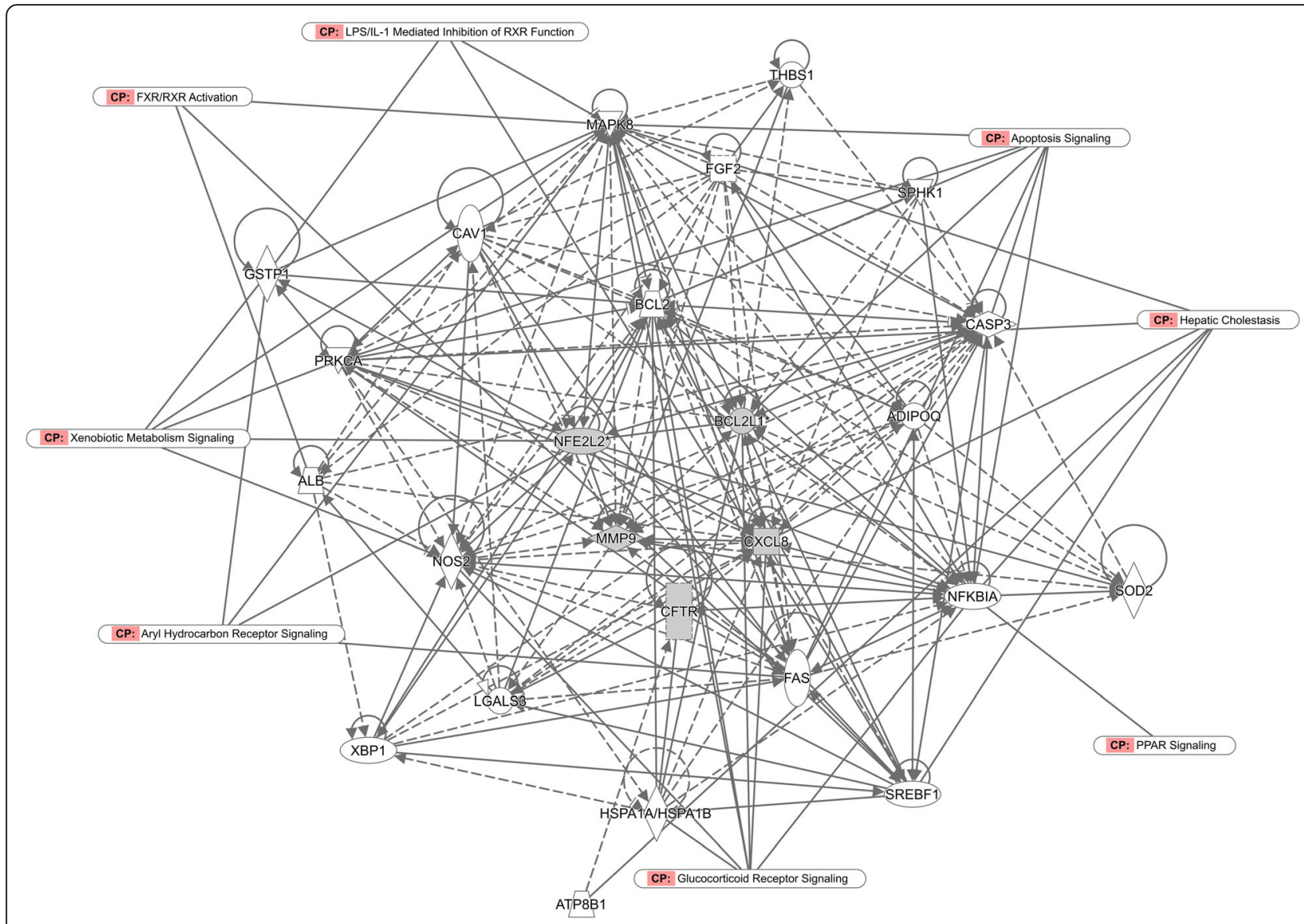

Fig. 6 The molecular network of both DILI and VOAAF

mechanism, the metabolic idiosyncratic hepatotoxicity mechanism, the mitochondrial damage mechanism, the biliary injury mechanism, signal transduction and liver cell death mechanism, the lipid peroxidation damage mechanism, the calcium balance and cell membrane damage mechanism [26-28]. The pathogenesis of DILI often involves toxic drugs or toxic metabolites, which causes immune reactions, or directly affects hepatocytes, eventually leading to clinical sign of hepatitis. Through IPA, it was found that the target proteins of VOAAF mainly involved the diseases and biological functions of inflammatory response, gene expression, organismal injury and abnormalities. Those are right consistent with the increase of alanine transaminase (ALT) and aspartate aminotransferase (AST) levels and the histopathologic changes of liver tissues, which have been well verified by researcher with animal experiments through oral administration of VOAAF to rats or mice [29-32].

The core analysis and the comparison analysis with the IPA software to the canonical pathway indicated that the target proteins of VOAAF and DILI genes both were involved with pathways of AHR signaling and LPS/IL-1 mediated inhibition of RXR function.
The AHR signaling is a nuclear receptor signaling, and it is mostly related with apoptosis, cell cycle regulation and xenobiotic metabolism. AHR is a ligand-activated transcription factor which locates in the cell cytoplasm, and it belongs to the superfamily of basic helix-loophelix per-arnt-sim homology domain protein subunits. AHR is activated by its related ligand after binding with it, and thereupon it translocates into cell nucleus to formulate the dimer complex which could bind with the corresponding deoxyribonucleic acid (DNA) sequence and enhances the expression of the cytochrome P450 (CYP450) with the AHR nuclear translocator [33]. Cytochrome P1A1 (CYP1A1) and cytochromeP1A2 (CYP1A2) could metabolise many compounds into toxic substance, such as the reactive oxygen species (ROS). Through this way, CYP1A1 and CYP1A2 could influence on the mitochondria, and lead to the decrease of the mitochondrial membrane potential $[34,35]$. The classic agonist of AHR, 2, 3, 7, 8-tetrachlorodibenzo-pdioxin (TCDD) could produce extra $\mathrm{H}_{2} \mathrm{O}_{2}$ in the primary hepatocytes of rats and mice. TCDD could down-regulate the superoxide dismutase (SOD), catalase (CAT), glutathione peroxidase (GSH-Px) in the primary 
rats hepatocytes and decrease the activity of the mitochondria complex as well $[34,36]$. AHR could bind DNA directly in the mitochondria of liver cells, to influence the expression of the mitochondrial proteome, and then lead to the change of the function of the mitochondria and induce the liver injury [37]. In our previous study, it was found that after the gavage of a certain amount of VOAAF to mice, a large amount of ROS would be generated, and the content of the malondialdehyde (MDA), oxidized glutathione (GSSG) in the liver tissues both increased obviously, while the antioxidation activity of the SOD and the content of the glutathione (GSH) decreased obviously [29, 32]. The mitochondria became swollen, and mitochondrial membrane potential decreased as well as the activity of $\mathrm{Na}^{+}-\mathrm{K}^{+}$-ATPase, $\mathrm{Ca}^{2+}$-ATPase and $\mathrm{Ca}^{2+}-\mathrm{Mg}^{2+}$-ATPase [38]. According to those results, it is proposed that the AHR signaling pathway could be the major one related to VOAAF-induced liver injury, and it mainly involves mitochondrial damage.

LPS/IL-1 mediated inhibition of RXR function is a nuclear receptor signaling. The top functions and diseases of it are lipid metabolism; molecular transport; small molecule biochemistry. Lipopolysaccharide (LPS), a major component of the outer membrane of gramnegative bacteria, potently stimulates host innate immune response. LPS binds the cysteine desulfhydrase 14 (CD14)/trithorax-like 4 (TRL4)/myeloid differentiation protein-2 (MD2) receptor complex, which promotes the secretion of pro-inflammatory cytokines like IL-1, tumor necrosis factor- $\alpha$ (TNF- $\alpha$ ) in various cells, especially in macrophages. Infection, inflammation and injury down-regulate the expression of hepatic genes involved in a variety of physiological processes, collectively known as the negative hepatic acute phase response (APR). Many of the repressed genes during $\mathrm{APR}$ are regulated by the nuclear hormone receptor retinoid $\mathrm{X}$ receptor $\alpha(\mathrm{RXR} \alpha)$. In response to proinflammatory cytokines signaling, $\mathrm{RXR} \alpha$ undergoes a cJun N-terminal Kinase (JNK) mediated, chromosome region maintenance 1 (CRM-1)-dependent nuclear export, leading to decreased nuclear $\mathrm{RXR} \alpha$ levels and reduced nuclear DNA binding and transcriptional activity. Reduction in the expression of hepatic transport proteins (ATP-binding cassette $(\mathrm{ABC})$, organic anion transporting polypeptide (OATP), multidrug resistance gene 1 (MDR1), $\mathrm{Na}^{+}$/taurocholate cotransporting polypeptide (NTCP)), metabolizing enzymes (CYP, glutathione transferase (GST), UDP-glycosyltransferase (UGT), SOD) and biosynthesis enzymes (CYP7A1), leads to impaired metabolism, transport and/or biosynthesis of lipid, cholesterol, bile acid and xenobiotics. A research indicated that the JNK pathway was closely related to the hepatic cells death [39]. Usually the activation of this pathway could be inhibited by the survival gene of nuclear factor kappa $\beta(\mathrm{NF}-\kappa \beta)$, so that the inhibition of the activation is transitory and non-toxic. However, the consistent activation of the pathway may lead to the cell death. The JNK pathway could be activated by many stressors, such as the ROS, ultraviolet (uv) light, receptor-interacting proteins [40], which lead to the cell death finally. Besides, the mitochondria is the major target of the JNK pathway. The consistent activation of JNK signaling pathway occurs in the mitochondria, affects the function of mitochondria and magnifies the oxidative stress, eventually leading to mitochondrial ailure and cell death. Therefore, the LPS/IL-1 mediated inhibition of RXR function involves in the signal transduction and liver cell death of DILI, indicating that the LPS/IL-1 mediated inhibition of RXR function may involve in the VOAAF-induced liver injury.

\section{Conclusions}

In this work, 2 molecular networks and their functions were analyzed through IPA. One network identified genes related to DILI, and the other identified VOAAF target proteins. Although the VOAAF could affect various DILI-related functions and pathways in various ways, the major affected functions and pathways were analyzed in this paper, and the effects on AHR signaling and LPS/IL-1 mediated inhibition of RXR function might be crucial for elucidating the molecular mechanism of VOAAF. In future work, experiments in vitro and in vivo will be performed to verify the results by IPA.

\section{Additional files}

Additional file 1: Table S1. DILI-related genes searched in PubChem. In the GenBank database 338 genes were identified involved in DILI. (DOCX $53 \mathrm{~kb}$ )

Additional file 2: Table S2. Target proteins of VOAAF searched in PubChem. Searched in the PubChem database, information on the drug targets of the VOAAF was listed. (DOCX $33 \mathrm{~kb}$ )

\section{Abbreviations}

ABC: ATP-binding cassette; AHR: Aryl hydrocarbon receptor; ALT: Alanine transaminase; APR: Acute phase response; AST: Aspartate aminotransferase; CAT: Catalase; CD14: Cysteine desulfhydrase 14; CNKI: China National Knowledge Infrastructure; CRM-1: Chromosome region maintenance 1; CYP1A1: Cytochrome P1A1; CYP1A2: cytochromep1a2; CYP450: Cytochrome P450; DILI: Drug-induced liver injury; DNA: Deoxyribonucleic acid;

FXR: Farnesoid X receptor; GI: Gen Info Identifier; GSH: Glutathione; GSHPx: Glutathione peroxidase; GSSG: Oxidized glutathione; GST: Glutathione transferase; IPA: Ingenuity Pathway Analysis; JNK: Jun N-terminal Kinase; LPS: Lipopolysaccharide; MD2: Myeloid differentiation protein-2; MDA: Malondialdehyde; MDR1: Multidrug resistance gene 1; NF-k $\beta$ : Nuclear factor kappa $\beta$; NTCP: $\mathrm{Na}^{+} /$taurocholate cotransporting polypeptide; OATP: Organic anion transporting polypeptide; PPAR: Peroxisome proliferator-activated receptor; PPI: Protein-protein interaction; PXR: Pregnane $X$ receptor; $R X R$ : Retinoid $X$ receptor; $R X R a$ : Retinoid $X$ receptor a; siRNAs: Small interfering RNAs; SOD: Superoxide dismutase; TCDD: 2, 3, 7, 8tetrachlorodibenzo-p-dioxin; TCM: Traditional Chinese medicine; TNFa: Tumor necrosis factor-a; TRL4: Trithorax-like 4; UGT: UDP- 
glycosyltransferase; uv: Ultraviolet; VOAAF: Volatile oils from Artemisiae Argyi Folium

\section{Acknowledgements}

Not applicable.

\section{Funding}

This research was supported by National Natural Science Foundation of China (No. 30901936, 81,473,688 and 81,673,979).

\section{Availability of data and materials}

The datasets generated and analyzed during the study are available from the corresponding author on reasonable request.

\section{Authors' contributions}

ZZ, MM and HL designed the research; SZ, LiaC, LinC, HD, YZ and YM analyzed and interpreted the data; $\mathrm{HL}$ and SZ drafted the manuscript; MM and ZZ revised the manuscript. All authors read and approved the final manuscript.

\section{Ethics approval and consent to participate}

Not applicable.

\section{Consent for publication}

Not applicable.

\section{Competing interests}

The authors declare that they have no competing interests.

\section{Publisher's Note}

Springer Nature remains neutral with regard to jurisdictional claims in published maps and institutional affiliations.

Received: 19 June 2017 Accepted: 7 November 2017 Published online: 16 November 2017

\section{References}

1. Luscombe NM, Greenbaum D, Gerstein M. What is bioinformatics? A proposed definition and overview of the field. Method Inform Med. 2001; 40(4):346-58.

2. Cheng $X$. The outlook of bioinformatics development. Chin Sci Technol Inf 2006:8:241-3. (in Chinese)

3. Zhang $C$. The current status and the prospect of bioinformatics. World Sci Technol Res Dev. 2000;22:17-20. (in Chinese)

4. Zhao N, Li J, Li L, Niu X, Jiang M, He X, Bian Z, Zhang G, Lu A. Molecular network-based analysis of guizhi-shaoyao-zhimu decoction, a TCM herbal formula, for treatment of diabetic peripheral neuropathy. Acta Pharmacol Sin. 2015;6:716-23

5. Gu P, Chen H. Modern bioinformatics meets traditional Chinese medicine. Brief Bioinform. 2014;6:984-1003. (in Chinese)

6. Barabási AL, Gulbahce N, Loscalzo J. Network medicine: a network-based approach to human disease. Nat Rev Genet. 2011;12:56-68.

7. Nanjing University of Traditional Chinese Medicine. Part I. In: Yu H, Liu S, editors. The grand dictionary of Chinese medicine. Shanghai: Shanghai Scientific \& Technical Publisher; 2015. p. 801-4. (in Chinese).

8. The Editorial Committee for Chinese Materia Medica, State Administration of Traditional Chinese Medicine of China. Part 7. In: Ying X, editor. Chinese Materia medical. Shanghai: Shanghai Scientific \& Technical Publisher; 1999. p. 668-75. (in Chinese)

9. State Pharmacopoeia Committee. Part I. In: Zhao Y, Fan Z, Huang K, Li Z, Gao Y, editors. Pharmacopoeia of the People's Republic of China. Beijing: Chinese Medical Science Press; 2015. p. 89. (in Chinese).

10. $\mathrm{Li} \mathrm{H}$. The pharmacological research progress and application development of the argy wormwood leaf. Prim J Chin Mater Medica. 2002;16:51-3. (in Chinese)

11. Jiang H, Hou AJ, Xiang ZX, Chen YX. Study on Artemisia argyi essential oil: anti-inflammatory, antianaphylatic and analgesic effects. J New Med. 2005; 15:36-9.

12. Ge YB, Wang ZG, Xiong Y, Huang XJ, Mei ZN, Hong ZG. Anti-inflammatory and blood stasis activities of essential oil extracted from Artemisia argyi leaf in animals. J Nat Med. 2016;70:1-8.

13. Huang HC, Wang HF, Yih KH, Chang LZ, Chang TM. Dual bioactivities of essential oil extracted from the leaves of Artemisia argyi as an antimelanogenic versus antioxidant agent and chemical composition analysis by GC/MS. Int J Mol Sci. 2012:13:14679-97.

14. Bao XL, Yuan HH, Wang CZ, Liu JJ, Lan MB. Antitumor and immunomodulatory activities of a polysaccharide from Artemisia argyi. Carbohydr Res. 2013;98:1236-43.

15. Zeb A, Sadiq A, Ullah F, Ahmad S, Ayaz M. Phytochemical and toxicological investigations of crude methanolic extracts, subsequent fractions and crude saponins of Isodon rugosus. Biol Res. 2014;47(1):1.

16. Ayaz M, Junaid M, Subhan F, Ullah F, Sadiq A, Ahmad S, Imran M, Kamal Z, Hussain S, Shah SM. Heavy metals analysis, phytochemical, phytotoxic and anthelmintic investigations of crude methanolic extract, subsequent fractions and crude saponins from Polygonum Hydropiper L. BMC Complement Altern Med. 2014;14(1):1.

17. Sun $R$, Huang $W$, Wang $H$. The research progress of the chemical constituents of the argy wormwood leaf related to efficacy and toxicity. Chin J Pharmacovigilance. 2009;6:676-9. (in Chinese)

18. Sun R, Li S. The research progress of the toxicity of the argy wormwood leaf based on the efficacy and material. J Shandong Univ Trad Chin Med. 2010; 34:86-8. (in Chinese)

19. Wang Y, Bolton E, Dracheva S, Karapetyan K, Shoemaker BA, Suzek TO, et al. An overview of the PubChem BioAssay resource. Nucleic Acids Res. 2010;38: D255-66.

20. Wu Y, Xu D, Wang Z, Wang $R$, Zhang $X$, Wang L. Progress on applying of bioinformatics in drug discovery. China J Bioinform. 2005; 3:81-92. (in Chinese)

21. Wu Y, Zhu H. Modernization research on bioinformatics and traditional Chinese medicine. Mod J Integr Trad Chin Western Med. 2004;13:1399-401. (in Chinese)

22. Shang H, Li S, Gao X. Multi-objective and fuzzy optimization of the different ratios of danshen (Salvia miltiorrhiza) to sanqi (Panax notoginseng) in composing formulas. J Beijing Univ Trad Chin Med. 2003;26:28-31. (in Chinese)

23. $\mathrm{Xu} \mathrm{J.} \mathrm{Recommendations} \mathrm{for} \mathrm{the} \mathrm{diagnosis} \mathrm{and} \mathrm{treatment} \mathrm{of} \mathrm{acute} \mathrm{drug-}$ induced liver injury (draft). Chin J Dig. 2007;27:765-7. (in Chinese)

24. Ren D, Deng C. Research advances in drug-induced liver injury. J Military Surg Southwest China. 2014;16:670-2. (in Chinese)

25. Shi Z, Wang W, Deng S. Clinical efficacy of liver lesion with the treatment of reduced glutathione and ademetionine. Acta Univ Med Anhui. 2014;49: 122-4. (in Chinese)

26. Xu X, Ji L, Lu L, Chen L, Xin Y, Xuan S. Research advances in the pathogenesis of drug-induced liver injury. J of clinical. Hepatology. 2016;2:382-5.

27. Xu X, Qu C. Mechanisms of drug-induced liver injury. Med Recapitulate. 2008;14:747-9.

28. Chen $Y$, Lyu $W$. The research development on the mechanism of traditional Chinese medicine drug-induced liver injury. J Basic Chin Med. 2015:11:1476-8. (in Chinese)

29. Liu H, Bai Y, Hong Y, Zhang X. Component analysis and acute hepatotoxicity of volatile oils from argy wormwood leaf extracted by different methods. J Chin Mater Med. 2010;35:1439-46. (in Chinese)

30. Huang W, Zhang Y, Wang H, Zhu L, Sun R. Experimental study on the "Dose-Time-Toxicity" relationship of single dose hepatotoxicity induced by different components from folium artemisiae argyi in mice. Chin J Pharmacovigilance. 2011;8:392-6. (in Chinese)

31. Huang W, Zhang $Y$, Wang $H$, Sun R. Experimental study on the "Dose-TimeToxicity" relationship of multi-dose hepatotoxicity induced by different components from folium artemisiae argyi in mice. Chin J Pharmacovigilance. 2011:8:397-400. (in Chinese)

32. Gong $Y$, Huang $W$, Qian $X$, Wang $H$, Zhang $Y$, Sun R. Experimental study on chronic toxicity in rats caused by different components of folium artemisiae argyi. Chin J Pharmacovigilance. 2011;8:401-6. (in Chinese)

33. Zhang B, Jing C, Li X, Wang J. Effect of 1-octyl-3-methylimidazolium bromide on the expressions of CYP1A1, CYP1A2, CYP3A4, and GSTP1, and the receptors AhR, ARNT, and PXR in HepG2 cells. Toxin Rev. 2015;34:161-7.

34. Aly HAA, Domenech O. Cytotoxicity and mitochondrial dysfunction of 2,3, 7, 8-tetrachlorodibenzo-p-dioxin (TCDD) in isolated rat hepatocytes. Toxicol Lett. 2009;191:79-87.

35. Senft AP, Dalton TP, Nebert DW, Genter MB, Puga A, Hutchinson RJ, Kerzee JK, Uno S, Shertzer HG. Mitochondrial reactive oxygen production is dependent on the aromatic hydrocarbon receptor. Free Radic Biol Med. 2002;33:1268-78.

36. Wang X, Li K, Liu L, Shi Q, Song $P$, Jian Z, et al. AHR promoter variant modulates its transcription and downstream effectors by allele-specific AHR- 
SP1 interaction functioning as a genetic marker for vitiligo. Sci Rep. 2015;5: 13542.

37. Hwang HJ, Dornbos P, Steidemann M, Dunivin TK, Rizzo M, LaPres JJ. Mitochondrial-targeted aryl hydrocarbon receptor and the impact of 2, 3, 7 , 8-tetrachlorodibenzo-p-dioxin on cellular respiration and the mitochondrial proteome. Toxicol Appl Pharmacol. 2016;304:121-32.

38. Liu H, Li T, Zhan S, Chen L, Chen L, Tang S. Effect and mechanism of acute liver injury induced by volatile oil from folium artemislae argyi in mice. Chin J Clin Pharmacol Ther. 2017;03:248-52. (in Chinese)

39. Ming $Y$, Liu $X$, Mao $Y$. The pathogenesis of drug-induced liver injury (DILI). J Pract Hepatol. 2014;17:657-60.

40. Ramachandran A, McGill MR, Xie Y, Ni H, Ding W, Jaeschke H. Receptor interacting protein kinase 3 is a critical early mediator of acetaminopheninduced hepatocyte necrosis in mice. Hepatology. 2013;58:2099-108.

Submit your next manuscript to BioMed Central and we will help you at every step:

- We accept pre-submission inquiries

- Our selector tool helps you to find the most relevant journal

- We provide round the clock customer support

- Convenient online submission

- Thorough peer review

- Inclusion in PubMed and all major indexing services

- Maximum visibility for your research

Submit your manuscript at www.biomedcentral.com/submit
Biomed Central 